\title{
The Application of Threshold Segmentation Algorithm in Loess Microstructure Image Analysis
}

\author{
Nian-dong Deng \\ Dept. of Geology and Environment Engineering \\ Xi'an University of Science and Technology \\ Xi'an, China \\ E-mail:gissearch@163.com \\ Wang Feng \\ Dept. of Geology and Environment Engineering \\ Xi'an University of Science and Technology \\ Xi'an, China
}

\author{
Wang Xiao \\ Dept. of Geology and Environment Engineering \\ Xi'an University of Science and Technology \\ Xi'an, China \\ Zhou Yang \\ Dept. of Geology and Environment Engineering \\ Xi'an University of Science and Technology \\ Xi'an, China \\ E-mail :hero-zhouyang@163.com
}

\begin{abstract}
The image research on loess microstructure which has profound influence on loess engineering mechanics properties is a new subject of geotechnical, the loess microstructure information and its section of the occurrence can be reflected via CT or electron microscope technology. The loess microstructure image was interpreted by the loess microstructure image processing system developed through $\mathrm{VC}++6.0$ platform. According to the different gray values of the different materials in the microstructure image, the image segmentation algorithm was used to obtain the binary segmentation which showed that the image contrast was enhanced, noise elimination and background purification effect were effective. This paper aims to expand the application category of microscopic image processing and explore research methods of loess microstructure, which has positive guiding significance on the analysis of loess engineering properties.
\end{abstract}

Keywords-Image Segmentation; Loess; Microstructure

\section{INTRODUCTION (HEADING 1)}

China has the world's largest loess covered area ${ }^{[1]}$, but Loess geological disasters which based on loess characteristics, supplemented by the impact of human activities and the frequent occurrence of extreme climate pose a serious safety problems on industrial, agriculture, construction and transportation and other fields and has seriously restricted the economic development in loess area. In addition to natural force and human activities and so on, loess disasters and its physical and mechanical properties of the internal is more inseparable, and the physical and mechanical properties depends heavily on its microstructure. To explore the relationship between microstructure of loess and loess collapse and macroscopic mechanical response, first on loess microstructure quantitative measurement, analysis and understanding of soil microstructure distribution, in order to further study of microstructure is how to influence the internal stress and the deformation and the distribution of the soil and the failure process and the regularity of soil .CT technology

This work is supported by the National Natural Science Foundation of China (41002101, 40972174), Scientific Research Program Funded by Shaan Xi Provincial Education Commission (Program NO.2010JK677). and electron microscopy as a high resolution capabilities of the nondestructive testing technology, already from the medical profession and the industry popularizes application to geotechnical engineering world, which offers a new approach for microscopic study of loess structure deformation failure mechanism under various load modes and loess microstructure model reconstruction .

Although microstructure data analysis there are many ways, but image analysis is still the basic work which must be carried [2], because loess microstructure image structure is complex, each medium has gray on the vagueness and uncertainty. In order to solve these weaknesses, accurately distinguish each medium, we need to carry out image segmentation treatment on loess microstructure image, so image segmentation is one of indispensable means of quantitative information to extract the specific parts of image. Therefore, the author according to the core idea and algorithm of a variety of image segmentation processing, realize a variety of segmentation functions of the microstructure image in the development of the image processing system program.

\section{Threshold SEgMENTATION Algorithm BRIEF INTRODUCTION}

Threshold segmentation is a common directly on the image segmentation algorithm, according to the image pixel gray value of different and decide, corresponding to a single target image, need to choose a threshold value, then the image is divided into two kinds of target and background, this is called single threshold segmentation, If the target image is complex, selecting multiple threshold value, can divided the target area and background of image into multiple, this is called many threshold segmentation, at the moment it is need to differentiate test results of image target, distinguish various image target region by unique identification. The advantages of threshold segmentation are obvious, low cost, simple realization. When the pixel gray value or other features of the target and background regions exist obvious differences, the algorithm 
can effectively realize the image segmentation. the key of threshold segmentation method is how to obtain a suitable threshold value, In recent years the methods are: the method of selection threshold by the maximum principle of relevance, based on image topological stable state method, gray level cooccurrence matrix method, the maximum entropy method and Valley value analysis method and so on. More cases, the selection of threshold will be integrated with two or more than two kinds of methods; it is also a trend of the development of image segmentation.

Gray threshold segmentation method is one of the most commonly used parallel Regional Technology, It is a kind of most application method in image segmentation. In fact, threshold segmentation method is actually the transformation from the input image $f$ to output image $g$.

$$
g(i, j)= \begin{cases}1 & f(i, j) \geq T \\ 0 & f(i, j)<T\end{cases}
$$

Thus, the key of threshold segmentation algorithm is to determine the threshold, if we can determine an appropriate threshold, the image can be accurately segmented.

The threshold is determined, we can parallel to compare the threshold with the pixel gray value and pixel segmentation of each pixel, the segmentation results are given directly image area. Threshold segmentation has the advantages of simple calculation, high efficiency of the algorithm, high speed. In this applications that emphasized on operational efficiency (such as for hardware implementation), it has been widely applied. Methods mainly include the optimal threshold method, minimum threshold value method, iterative threshold method and so on.

\section{IMAGE PROCESSING OF LOESS MICROSTRUCTURE}

\section{A. grayscale image}

The best application software which is compatibility with Windows BMP format, the image format used in this test system is unified for the BMP format. During the binary morphological processing, we must ensure that the image is a binary image. First of all, we should transform the image to grayscale, grayscale only have brightness information and no color information, as people usually see photographs which the brightness from dark to black and white, its brightness change is continuous. Therefore we have to quantify the brightness value if we want to express grayscale images, Brightness value usually divided into 0 to 255levels and 0 is the darkest (black) and 255 of the brightest (white). This test using image processing system which is developed by $\mathrm{VC}+$ +6.0 to convert loess image into 256 grayscale image, as shown in Figure 3 (a) below.

\section{B. Image restoration}

During the conversion process, it is inevitable that there will be lack of information and noise increase, resulting in a decline in image quality. In order to achieve the desired parameters, before image analysis we must repair process. Repair methods include image restoration and image enhancement, both of them are aim to improve image quality, but the way to achievement is very different. Image restoration use the priori knowledge of the degradation process restores true colors of the degraded image; image enhancement reduces the noise in the image to improve the contrast between target and background and to emphasize or suppress certain details in the image. From the point of view of image quality assessment, image restoration could improve the intelligibility of the image; image enhancement aims to improve the quality of the visual sense ${ }^{[2]}$.

\section{Threshold segmentation}

\section{1) Gray histogram}

A histogram is the function of gray level, it describing the statistical results of the frequency of gray levels in an image. By analysis histogram, we could determine the threshold value of the binarization processing; it is the optimum threshold for image segmentation in order to extract the important feature information of the micro structure image the Lishi histogram shown in Figure 1.

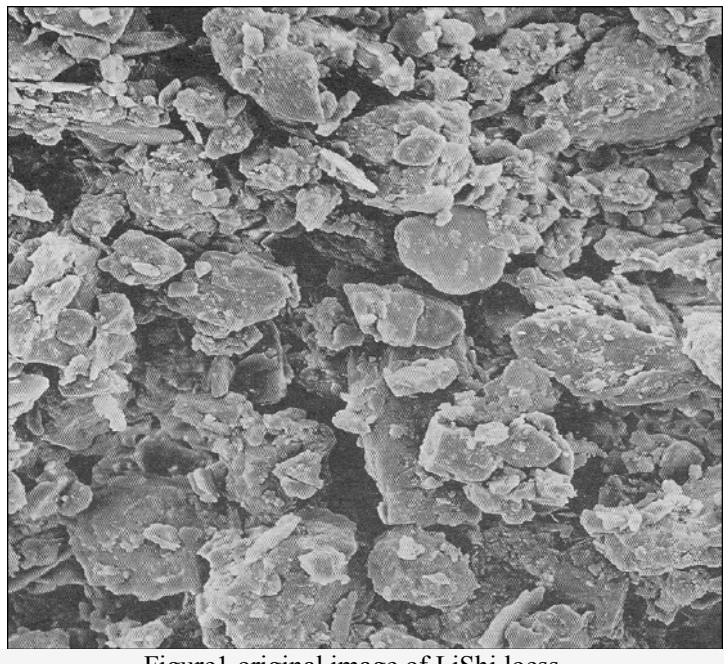

Figure1 original image of LiShi loess

\section{2) Threshold segmentation}

The choice of the threshold is needed to determine based on the specific questions. This test determined threshold by using the minimum point threshold. we could consider histogram envelope is a curve by minimum point threshold method and to strike a curve minimum value as the threshold value of the image segmentation. Therefore Minimum point should satisfy the following relationship as shown in Formula 3-1

$$
p^{\prime}(z)=0 \text { 且 } p^{\prime \prime}(z)>0
$$

Choosing 100 is the optimal threshold while we are analyzing the histogram of the Lishi Loess micro-structural image. In addition, by Otsu threshold image segmentation, image shown in Figure 2.

By image processing, we can clearly see the characteristics of the Lishi loess cross section nature. Particle morphology such as columnar, rod, rounded and flaky. Grains are contacted by surface-surface, so the structural is steady and has small pore. The cement is filling in the pore and set intergranular, they are partially cemented and to be a mosaic 
structure of micro-porous cementation. We can find a clear vertical fracture central in the image, which shows the development of the vertical joints in the loess.

Using Otsu threshold segmentation on Malan loess, the image shown in Figure 3.
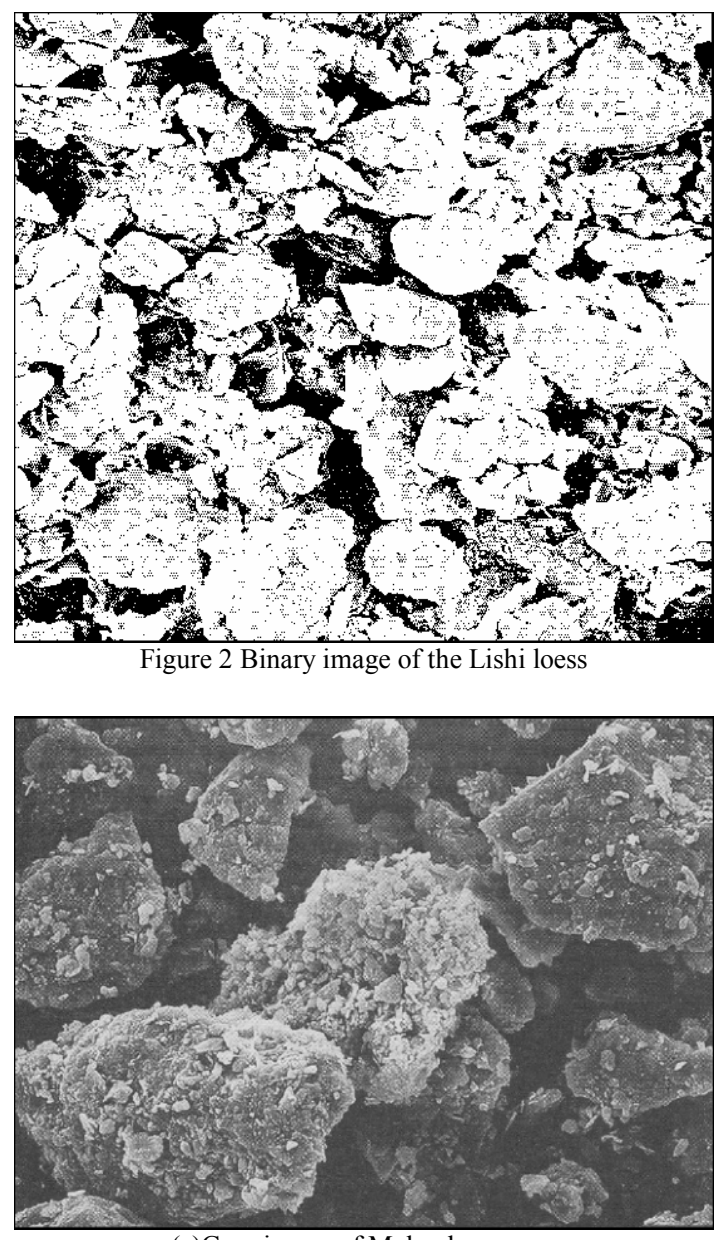

(a)Gray image of Malan loess

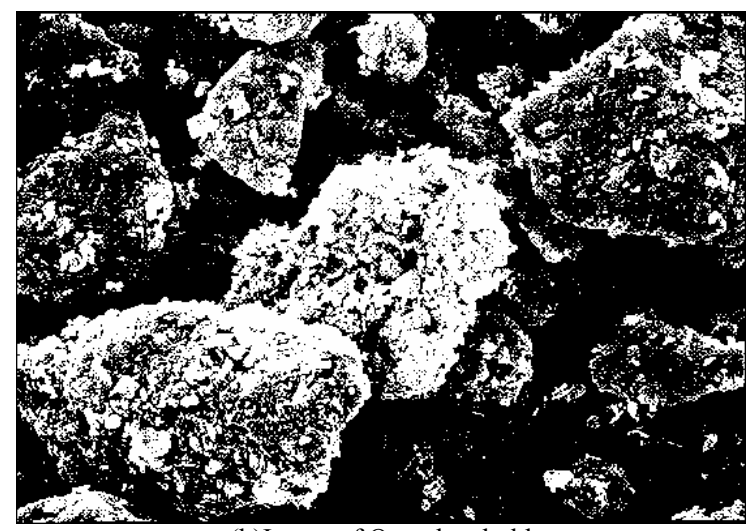

(b)Image of Otsu threshold

Figure 3 Gray image and Otsu threshold image

Through image processing, contrast enhancement between the loess internal particles and pore. Coarse the skeleton more obvious of undisturbed Malan loess, it has high porosity and the coarse skeleton often is made of the aggregates of clay particles, so the soil osteoporosis, it has bracket macro-porous structure, there are fissures in the structure. Therefore, the structure is extremely unstable, once in contact with water or compaction, the polymer backbone is easily damaged.

\section{RESULT}

This paper achieve a combination of loess microstructure image and image processing technology, analysis of the characteristics of loess samples, qualitative analysis of the nature of its micro-structure. If we compare the image of loess micro-structure which is in a group of the same layer in different stress in the practical application, we could get the trend of loess microstructure under stress and This method provides information and theoretical basis for the study of loess mechanism, catastrophic evolution and Engineering Properties.

\section{REFERENCES}

[1] WANG Nian-qin,ZHANG Zhuo-yuan, Study of loess landslides[M]. LanZhou University Press. March 2005 edition

[2] HE Dong-jian,GENG Nan,ZHANG Yi-kuan, Digital Image Processing [M]. Xi'an University of Electronic Science and Technology Press. May 2008 Second Edition 